\title{
¿Que es calidad total ?
}

\section{Luis Lozano Cortijo*}

\section{Antecedentes}

La preocupación por la calidad no es nueva. En la Antigüedad eran los mismos campesinos o artesanos quienes producían o fabricaban los productos y controlaban la calidad de su trabajo, siendo a la vez productores y consumidores.

Con el desarrollo de la sociedad mercantil se van separando el productor y consumidor para dar paso al intermediario.

Posteriormente la aparición de la tecnología moderna en la Revolución Industrial cambia la forma de producción, generando una división del trabajo, la especialización y la creación de la línea de ensamble. Se da paso a una nueva forma de vida en la sociedad y a una transformación de las organizaciones.

Con el crecimiento y desarrollo de la organización industrial surge una nueva figura que vendría a reemplazar al antiguo capataz en sus funciones de control: el inspector, encargado inicialmente de seleccionar los productos en buenos y defectuosos. Esta etapa se caracterizó por la inspección ejercida sobre el producto final y por la aparición del sistema de producción en línea creado por Henry Ford.

El control de calidad moderno o control de calidad estadístico, comenzó en los años 30.
La utilización estadística como la herramiento que mide la variación de los procesos, propición el cambio del modelo de control "estático o correctivo" a uno "dinámico, preventivo y evaluativo".

La nueva revolución tecnológica, representada por el invento de nuevos materiales, la microelectrónica, la biotecnología y la cibernética, trajo consigo un cambio de concebir la calidad del producto en todas sus fases de transformación, ya no desde la óptica del productor, sino con la óptica puesta en el consumidor. Es precisamente en este momento cuando el concepto de CONTROL TOTAL DE CALIDAD se convierte en una filosofía que tiene como propósito la satisfacción del consumidor.

El doctor Armand V. Felgenbaum en Mayo de 1957 da origen al concepto del control total de calidad definiéndolo como: "un sistema eficaz para integrar los esfuerzos en materia de desarrollo, mantenimiento y mejoramiento de la calidad, realizados por los diversos grupos en una organización, de modo que sea posible producir bienes y servicios a los niveles más económicos y que sean compatibles con la plena satisfacción de los clientes" * Médico Maestro en Administración de Salud Docente del Dpto. de Salud Pública y del
centro de Salud Pública de la Universidad Peruana Cayetano Heredia. Lima, Perú. 


\section{La irrupción de la calidad total en el mundo actual}

El desarrollo de la concepción de control total de calidad hasta la década de 1970 no fue predominante, debido a que la administración se fue dando en mercados crecientes. Fue mayor énfasis en el control estadístico de calidad de productos.

A mediados de la década del '70 los mercados estancados y los mercados decrecientes en el '80 generaron la ruptura con los enfoques tradicionales de administración, emergiendo un conjunto de enfoques que bajo la denominación de administración estratégica van reflexionando sobre la calidad de bienes y servicios, particularmente a raíz del éxito de la administración japonesa.

Control Total de Calidad, Calidad Total, Sistema de Calidad Total en el Trabajo, son términos utilizados para a un enfoque administrativo o estilo de gestión, cuyos representantes más conspicuos son: E. Edwards Deming, Joseph Juran, Philip B. Crosby y Kaoru Ishikawa.

\section{W. Edwards Deming:}

El Dr. Deming es el estadístico más famoso por haber logrado encaminar la industria japonesa a ocupar el primer lugar en calidad del mundo. En 1950 viajó a Japón, con el fin de realizar un censo de población y fue invitado a dictar conferencias a los principales dirigentes industriales sobre el control estadístico de la calidad.

Deming afirma: "El $94 \%$ de los problemas de calidad son responsabilidad de la alta gerencia" y señala que es un deber de ésta ayudar a las personas a trabajar con más astucia y no a trabajar más". Las empresas que desean cumplir metas y objetivos de muy corto plazo en el campo económico, político o social pueden poner en peligro la permanencia de la organización en el largo plazo.

La inspección según el autor, bien sea de los materiales, o insumos que entran o de los bienes que salen, es tardía, ineficiente y costosa. "La inspección no mejora la calidad ni la garantiza".
Los siguientes 14 principios que debe cumplir la gerencia constituyen la columna vertebral del enfoque de Deming:

1. Mejoramiento permanente del producto $\mathrm{y}$ del servicio.

2. No seguir conviviendo con niveles aceptables de errores, retrasos y materiales defectuosos.

3. Abandonar la dependencia de la inspección masiva.

4. Abolir la práctica de hacer los negocios solamente basados en el precio, "se debe comprar calidad".

5. Detectar los problemas mejorando constantemente el sistema.

6. Entrenar a los trabajadores, enseñándoles como hacer mejor el trabajo.

7. Instituir métodos modernos de supervisión del personal de producción, haciendo que la responsabilidad de los empleados cambie de las cifras a la calidad.

8. Erradicar el temor para que todo el mundo pueda trabajar eficientemente en la empresa.

9. Derribar las barreras que existen entre los departamentos.

10. Eliminar metas numéricas, lemas $\mathrm{y}$ slogans para la fuerza laboral.

11. Eliminar las cuotas numéricas. En lugar de definir niveles de productividad es necesario precisar los niveles de calidad.

12. Derribar los obstáculos que impiden hacer bien un trabajo.

13. Instituir un vigoroso programa de capacitación y reentrenamiento.

14. Tomar medidas para la transformación

\section{Joseph Juran:}

Al señor Juran al igual que Deming, se le debe gran parte del éxito del control de calidad en el Japón. Fue invitado por la unión de científicos e ingenieros japoneses (UCIJ) en 1954 para dictar seminarios a gerentes altos y medios. Las charlas del señor Juran crearon un nuevo ambiente entre los empresarios japoneses, en el cual se reconoció al control de calidad como un instrumento de gran utilidad para la gerencia. 
De acuerdo con Juran existen dos clases de calidad: La adecuada para utilización o uso" y "conformidad con especificaciones". Es decir que un producto puede estar fabricado de acuerdo con las especificaciones técnicas, pero su uso puede llegar a representar un grave riesgo para la población.

En los años 40 Juran afirmó que los aspectos técnicos del contro de calidad estaban bien definidos y revisados, pero que las empresas no sabían administrarlos. En su opinión, menos del $20 \%$ de los problemas de calidad se deben a los trabajadores y el resto es atribuible a la gerencia".

Juran afirmaba que al igual que algunos gerentes requieren de entrenamiento y capacitación en finanzas, todos ellos debían capacitarse en calidad a fin de conocer, manejar y controlar la presentación de proyectos de mejoramiento por parte del personal de la organización.

El mismo autor "está a favor de los círculos de calidad porque mejoran la comunicación entre la gerencia y los empleados".

Para Juran representa un peligro que la empresa dependa de una sola fuente de suministro cuando se trata de compras importantes (materia prima o componentes). Según él, "en compras importantes es sano tener fuentes múltiples de suministro. Una sóla fuente puede fácilmente descuidar su función competitiva de calidad, costo y servicio".

Las siguientes son las 10 etapas claves para el mejoramiento de la calidad:

1. Crear conciencia sobre la necesidad y oportunidad de mejoramiento.

2. Fijar metas para el mejoramiento.

3. Organizar la obtención de metas.

4. Establecer programas de capacitación.

5. Ejecutar proyectos para solucionar problemas.

6. Informar sobre los progresos.

$7 . \quad$ Dar reconocimiento.

8. Comunicar resultados.

9. Llevar un registro.

10. Mantener el "impulso" generado por el programa de mejoramiento.

\section{Philip b. Crosby:}

El señor Crosby, ha sido uno de los principales promotores del concepto de calidad durante más de 36 años. Su gran experiencia dentro del mundo empresarial como ex-vicepresidente de ITT, consultor y catedrático en administración y presidente de la firma "Philip Crosby Associataes, Inc." lo consagra como una de las mayores autoridades en el campo del control de calidad a nivel internacional.

"Todo el mundo está en favor de la calidad, nadie está en contra de ella, sin embargo rara vez se da por sí misma".

La calidad no es algo para controlar solamente, es necesario crearla, fabricarla, asegurarla y mejorarla permanentemente. Para lograr el mejoramiento de la calidad se requiere del compromiso y el esfuerzo de todos los miembros de una organización.

El "primer paso" para mejorar la calidad es obtener el compromiso de la alta dirección.

La calidad se debe ver positivamente como una forma de mejoramiento continuo en todos los niveles de la organización. No puede ser considerada como algo de exclusiva responsabilidad del personal técnico, requiere el liderazgo, el respaldo y la motivación de la alta gerencia y de los mandos medios.

Según Crosby, "todo trabajo es un proceso". Todo trabajo que realizamos es un proceso, es decir una serie de acciones que producen un resultado. Estos resultados son los productos (bienes o servicios) que satisfacen las necesidades y deseos de los clientes.

Para cumplir con las expectativas de los clientes se necesita primero identificar sus requisitos.

Crosby afirma, "el conocer y entender los requisitos de nuestro trabajo nos ayuda a satisfacer las necesidades de los clientes y a prevenir problemas".

Los cuatro principios absolutos de la calidad son:

1. Definición: ¿Cómo definimos Calidad?

2. Sistema: ¿Cómo podemos lograr la Calidad?

3. Estandar de realización: ¿Qué estandar de desempeño utilizamos?

4. Medición: ¿Cómo podemos medir la Calidad?

Finalmente, Crosby define la Calidad como: "el cumplimiento de los requisitos y no como algo bueno o aceptable".

Rev Med Hered 9(1), 1998 30 


\section{Kauro Ishikawa:}

En 1939 el Dr. Ishikawa se graduó en química aplicada de la Universidad de Tokio. Siendo profesor de ingeniería en esta Universidad, comprendió la importancia de los métodos estadísticos como herramienta en el campo de la calidad industrial. Desde 1949 ha ayudado a muchas compañías japonesas y extranjeras a alcanzar niveles destacados mediante la aplicación del control de calidad.

El control de calidad japonés en esencia se puede considerar como una revolución en el pensamiento de la gerencia. Según la definición del propio Ishikawa: "practicar el control de calidad es desarrollar, diseñar, manufacturar y mantener un producto de calidad que sea el más económico, el más útil y siempre satisfactorio para el consumidor".

La esencia misma del control de calidad es la GARANTIA DE CALIDAD lo que significa que calidad del producto o servicio se debe asegurar desde sus primeras etapas de desarrollo. Lo anterior, unido al concepto de la participación de todos los miembros de la organización es lo que ha dado origen al llamado "control de calidad de toda la empresa".

Las principales características del control de calidad japonés se reúnen en los siguientes seis puntos:

1. Control de calidad en toda la empresa, participación de todas las divisiones y sus empleados.

2. Educación y capacitación en control de calidad.

3. Actividades de círculos de calidad.

4. Auditoría de control de calidad.

5. Utilización de métodos estadísticos.

6. Actividades de promoción del control de calidad a la escala nacional.

A diferencia del enfoque norteamericano propuesto por Fegelbaum en donde el control de calidad debe estar en manos de especialistas respaldados en una función gerencial bien organizada, la modalidad japonesa insiste en que todas las divisiones y todos los empleados de una compañía deben participar en el estudio y promoción del control de calidad a través de la operacionalización de los siguientes postulados básicos:

1. Primero la calidad: no las utilidades a corto plazo.

2. Orientación hacia el consumidor. Es decir, pensar desde el punto de vista de los demás.

3. El proceso siguiente es su cliente: se deben derribar la barreras del seccionalismo.

4. Utilizar datos $\mathrm{y}$ números en las presentaciones, emplear métodos estadísticos.
5. Respeto a la Humanidad como filosofía administrativa, mediante el empleo de una administración participativa.

6. Administración interfuncional.

Las principales ventajas de la aplicación del control de calidad en las empresas generan los siguientes efectos en cadena. "Al aumentar la calidad de aceptación, paulatinamente disminuirán los defectos de los productos y aumentará el porcentaje de piezas de "paso-directo". Disminuirá el número de rechazos, en la correción de piezas. Esto dará como resultado una mayor economía de costos, acompañada de una productividad más alta, lo cual se verá reflejado en el aumento de las utilidades".

\section{Algunas definiciones sobre calidad \\ Vale la pena efectuar una muy breve investigación acerca de las definiciones que ha tenido la palabra calidad. \\ Juran sostiene que la palabra calidad tiene dos significados importantes: \\ 1. Aquellas características del producto que 2. La ausencia de deficiencias.}

Un término general que cubre los dos significados es "adecuación al uso".

Juran también utiliza los conceptos de:

CALIDAD GRANDE: para designar un concepto general de la calidad el el cual "clientes" incluye a todas las personas impactadas; "producto" incluye bienes y servicios; "procesos" incluye procesos empresariales y auxiliares.

CALIDAD PEQUEÑA: para designar un ámbito reducido de la calidad, limitada a compradores, artículos fabricados y procesos fabriles.

Deming, manteniendo su postura característica, no define a la calidad y se limita textualmente a decir:

"Repetimos aquí lo dicho en la página 4 (de su obra citada) de que la calidad comienza con la idea que es fijada por la dirección. La idea debe traducirse en planes, especificaciones, ensayos, en un intento de hacer llegar al consumidor la calidad deseada, todo lo cual es responsabilidad de la dirección".

Rev Med Hered 9(1), 1998 
Ishikawa aporta la siguiente definición:

"En su interpretación más estrecha, calidad significa calidad del producto. En su interpretación más amplia, calidad significa calidad del trabajo, calidad del servicio, calidad de la información, calidad del proceso, calidad de la división, calidad de las personas incluyendo a los trabajadores, ingenieros, gerentes y ejecutivos, calidad del sistema, calidad de la empresa, calidad de los objetivos, etcétera. Nuestro enfoque básico es controlar la calidad en todas sus manifestaciones" (op.cit, pág. 4).

Crosby, ofrece una muy numerosa gama de definiciones de la calidad entre las cuales elegimos las siguientes:

"Calidad significa cumplir con las especificaciones. No tener calidad es no cumplir con las especificaciones".

"El propósito de la calidad no es acomodar lo que está mal. Consiste en eliminar todo aquello que está mal y en evitar que se repitan tales situaciones".

"La calidad debe definirse: cómo cumplir con los requisitos".

Feigenbaum, define la calidad así:

"Un sistema eficaz para integrar los esfuerzos de mejora de la gestión de los distintos grupos de la organización para proporcionar productos y servicios en niveles que permiten la satisfacción del cliente.

Ginebra y Arana, proporcionan la siguiente definición:
"Acumulación de experiencias satisfactorias repetidas (obviamente, tanto por parte del productor del bien o des servicio o del cliente de los mismos)" (Dirección por servicios, 1991, McGraw-Hill, México).

Preferimos no elegir una de las definiciones anunciadas ya que, fundamentalmente, coinciden en lo que persiguen: un producto o servicio que satisfaga las expectativas del cliente/consumidor por su relación calidad/costo y servicio.

\section{Los protagonistas de la calidad total}

Riccardo Riccardi sistematiza los enfoques de Calidad Total de la siguiente manera, en la cual plantea su propio enfoque de calidad total dando énfasis en el factor humano y la Calidad de Vida del Trabajo (CVT) indisolublemente junto con el Sistema de Garantía de Calidad productivístico (SGC) (Ver cuadro):

Asimismo, R. Riccardi sistematiza los aspectos básicos comprendidos en los distintos enfoques de CALIDAD TOTAL en cuatro (4) elementos:

\section{El cliente "rey"}

Noción de cliente externo (cliente, proveedor), cliente interno (entre las miembros de la organización) en la satisfacción de necesidades y requerimientos.

\begin{tabular}{|c|c|c|c|c|}
\hline MOOELO & Deming & Jurdin & Crosby & Riceardi \\
\hline OB.JETIVO & Calidad & Calidad & Calidad & Calidad \\
\hline $\begin{array}{l}\text { LA ESENCIA DEL } \\
\text { CAMBIO ES: }\end{array}$ & $\begin{array}{l}\text { Proceso de } \\
\text { manufectura }\end{array}$ & $\begin{array}{l}\text { Producto y } \\
\text { uso }\end{array}$ & La organización & El mercado \\
\hline $\begin{array}{l}\text { EL CAMBIO SE } \\
\text { REALIZA POA: }\end{array}$ & $\begin{array}{l}\text { Control estadístico } \\
\text { del proceso }\end{array}$ & $\begin{array}{l}\text { Diseno y control } \\
\text { de especiticaciones }\end{array}$ & $\begin{array}{l}\text { Cambio de actitudes y } \\
\text { mayor produclividad }\end{array}$ & $\begin{array}{l}\text { Auditoría clemte y } \\
\text { rekacion cliente'provedor }\end{array}$ \\
\hline $\begin{array}{l}\text { ABARCA } \\
\text { ESENCIALMENTE: }\end{array}$ & Cadena vertical & Cadens vertical & $\begin{array}{l}\text { Orgarización inicial } \\
\text { en la alta direcxión }\end{array}$ & $\begin{array}{l}S G C \text { y CVT on toda ba } \\
\text { organización autocoentrol } \\
\text { y autogestión }\end{array}$ \\
\hline $\begin{array}{l}\text { EL PITMO DE } \\
\text { CAMBIO ES: }\end{array}$ & Gradual $y \sin$ limstes & $\begin{array}{l}\text { Gradual y acepta } \\
\text { tolerancias }\end{array}$ & $\begin{array}{l}\text { Actitud hacerio bien de } \\
\text { entrada. Cero defectos }\end{array}$ & $\begin{array}{l}\text { Proceso permanente de } \\
\text { cambio debido al } \\
\text { mercado cliente }\end{array}$ \\
\hline HaY COSTO: & si & $\$$ & $\begin{array}{l}\text { No. Se compensa por } \\
\text { aborro en anticalistad }\end{array}$ & si \\
\hline $\begin{array}{l}\text { EL CAMBIO SE } \\
\text { INSTRUMAENTA EN: }\end{array}$ & $\begin{array}{l}\text { Un programa de mejora } \\
\text { permanemete }\end{array}$ & Un programa & Modo internitents & $\begin{array}{l}\text { Programas de proceso } \\
\text { permanente }\end{array}$ \\
\hline $\begin{array}{l}\text { EN CUANTO A } \\
\text { LOS RECURSOS } \\
\text { HUMANOS: }\end{array}$ & $\begin{array}{l}\text { Ensefiar. Dar } \\
\text { horramiontas. No motas } \\
\text { muméricas. No arengas. }\end{array}$ & $\begin{array}{l}\text { Medir por casto de no } \\
\text { colidad. }\end{array}$ & $\begin{array}{l}\text { Animación. } \\
\text { Motivación. } \\
\text { Sí a las arengas. }\end{array}$ & $\begin{array}{l}\text { Involucrados en ta } \\
\text { acoión comprometida. }\end{array}$ \\
\hline $\begin{array}{l}\text { RELACION CALLOAD } \\
\text { PRODUCTO: }\end{array}$ & Juntos. & & & Van juntos. \\
\hline SGCICVT & & & & $\begin{array}{l}\text { Indisolubiemento } \\
\text { juntos. }\end{array}$ \\
\hline
\end{tabular}




\section{La lucha contra el error}

Superar los standars de la performance actual y acercarse todo lo posible al 0 (cero) defectos.

\section{El trabajo en equipo}

Trabajo en equipo significa "toda la institución", poner a todo el personal de la institución a trabajar para conseguir la transformación. Laa transformación es tarea de todos.

\section{La constante capacitacion}

Analizando en profundidad el proceso de cambio que conduce a la empresa hacia la cultura de calidad, se evidencia de inmediato que la verdadera protagonista es la capacitación.

Una formación para la estrategia de calidad de la organización.

Una formación para la relación cliente-proveedor

Una formación para el trabajo en equipo

\section{Asuntos pendientes de la calidad total en salud}

En el ámbito de los servicios de salud, la apertura hacia las mayores demandas de la participación ciudadana en las decisiones que le afectan directamente, la salud como derecho y de necesidad básica por un lado, la escasez de los recursos, el incremento de los costos de la atención y la incorporación en el mercado de los bienes y servicios en salud, plantean el reto que en salud no se puede hablar de calidad sin tomar en cuenta la equidad.

Revisemos entonces, los asuntos pendientes para el logro de la calidad total en salud:

\section{Calidad y Equidad:}

El "cliente" (población, usuario o paciente) no es el "rey": debido a que el mercado es imperfecto en salud, tanto por los niveles de información técnica que la población o el paciente no dominan para decidir oportunamente, como por los costos de la atención que no garantizan a quien no pueda pagar los servicios, el disponer de calidad en la atención y por su carácter de necesidad básica en la que hay pocas opciones para elegir.

Los estandares de calidad de la atención: es un asunto por resolver la real satisfacción de la población o del paciente de la atención en salud, que va desde los estandares "objetivos" como son la capacidad técnica instalada en los servicios hasta los estandares "subjetivos" de la atención.

\section{Calidad de Vida del trabajo:}

El trabajo tayloriano y burocrático en salud: La fuerte influencia de las escuelas tradicionales en salud, dificultan el cambio de la cultura organizacional.

Ejemplos del enfoque de Calidad Total en un servicio de salud:

Efectividad y satisfación de la población y del personal de salud:

"En el consultorio externo de ginecología de un hospital, los médicos están insatisfechos porque gastan la mayor parte de su tiempo atendiendo pacientes con leucorrea. Un estudio simple de demanda muestra que ésta es la primera causa de atención y que la concentración media de consultas para pacientes que recibieron tratamiento es de cuatro. Se inicia una discusión con el equipo de médicos sobre la rutina de la atención: anamnesis, examen clínico y recolección de muestra preventiva en la primera consulta; análisis del examen y prescripción en la segunda consulta; evaluación de la respuesta y mejoría clínica y eventual solicitud de nuevo preventivo en la tercera consulta. El responsable de laboratorio de patología clínica presente en la reunión dice que el volumen de examenes preventivos está sobrecargando su servicio y que los médicos podrían contar con la lámina al fresco como recurso más apropiado para el diagnóstico etiológico de la leucorrea.

Los médicos afirman que no tienen cómo hacer ese examen porque no disponen de microscopio en el consultorio y muchos de ellos dijeron que ya se olvidaron cómo hacerlo. El patólogo considera que es mucho mas eficiente destacar un técnico en el consultorio con un microscopio que realizar tantos preventivos caros, inespecíficos para ese fin e innecesarios. Los médicos se interesan por el procedimiento que les permite ya en la primera consulta diagnosticar y prescribir tratamiento a los pacientes y que, en menos de un mes, se sienten ya preparados para realizar ellos mismos el examen, prescindiendo del técnico."

El usuario insatisfecho y la correción de errores: 
"Al final de la tarde el Dr. Sergio Z. analiza las historias de los pacientes que no acudieron a la consulta del ambulatorio. Entre ellas está la de Doña María, de 42 años, asintomática, que había acudido al servicio para hacerse un examen preventivo de cáncer de cérvico uterino. El informe del examen muestra carcinoma in situ. El Dr. Sergio llama a la enfermera responsable y solicita que la paciente sea vuelta a convocar. La visitadora domiciliara no consigue la casa de doña María porque la dirección está incompleta. El funcionario administrativo responsable por el llenado de la ficha de identificación dijo que cumplió su tarea, llenando en la ficha las informaciones que la paciente le dió".

"Llama la atención de la auxiliar que da las citas para control pre natal una señora que se aproxima con el retrato de un niño pegado a su camiseta. La señora pide su cita y se va. En el día fijado, con el mismo retrato en su ropa, aparece la señora por el ambulatorio pero dice que desea postergar su consulta. Y así sucesivamente varias veces regresó la señora, ya identificada como la "loca del retrato de la camiseta que sólo posterga la consulta". En una tarde el Dr. Pedro R. se aproximó a ella y le preguntó: ¿Por qué siempre viene al día fijado pero sólo para postergarla? Llorando ella le dice que aquel retrato es de su hijito, que se murió por una causa que ella desconoce y afirma que mientras no sepa por qué murió, no podrá conseguir cuidar de ese nuevo niño que lleva en el vientre. El médico promete solicitar la información al hospital en que ocurrió el deceso. Así lo hizo y le comunica a la madre las condiciones y el por qué de la muerte del niño. La paciente le agradece y le dice que que ahora sí podrá cuidar de este nuevo hijo. En ese momento quien se emociona es el médico".

El "capacitacionismo" vs. educación permanente:

"Un estudio de evaluación de la asistencia perinatal en instituciones públicas de una región metropolitana, reveló que las maternidades $\mathrm{A}, \mathrm{B}$ y $\mathrm{C}$ presentaban elevados indices de muerte neonatal por hipoxia y que el $70 \%$ de los bebés nacidos bañados en meconio no fueron aspirados en la sala de parto. El coordinador regional solicita de inmediato la programación de un curso de hipoxia perinatal. Los directores de maternidad reciben un oficio para que indique los profesionales que irán al curso y para asistir a una reunión con el coordinador. En ella se discute el problema y aparecen los siguientes elementos: en la maternidad A el equipo de aspiración no está funcionando adecuadamente; en la maternidad B no hay recursos humanos suficientes para la realización del procedimiento, y en la maternidad $\mathrm{C}$ hay equipos y personal suficientes."

4. La necesidad de desarrollar nuevos elementos y adaptar los aportes de los distintos enfoques de administración estratégica:

Es importante que se analicen, y se re-elaboren los aportes de las distintas escuelas de administración estratégica en el campo de la salud y no simplemente en una adaptación. En este sentido, creemos pertinente citar la siguiente reflexión de Riccardi sobre la Calidad Total como moda:

" La "excelencia" se constituyó en el slogan panacea en los comienzos de la década del 80 y nos suministró durante un periodo relativamente corto, la ilusión de que habíamos encontrado la fórmula del éxito.

No ha sido realmente así. En los comienzos en la década del 90, varias de las empresas calificadas de 'excelentes', han sufrido pérdidas, han caído en sus posiciones y, si bien no han desaparecido, luchan por sobrevivir bien lejos del perfil de excelencia que les atribuyó.

En la ola "excelente" hicieron buenos negocios los escritores-consultores-conferencistas y "plagiarios" que aparecieron a escala intercontinental con una rapidez que provoca suspicacia en cuanto a la solidez de sus conocimientos en el tema.

Hoy la excelencia no es todavía una mala palabra, pero se acerca el momento en que será repudiada $\mathrm{y}$ reemplazada por otra más de moda.

Algo por el estilo amenaza ocurrir con la "calidad", que de pronto aparece en el escenario del management y promete un aluvión de escritoresconsultores-conferencistas (por brevedad los indicaremos con la sigla ECC) que esperan haber encontrado su nuevo "El Dorado" en los meandros de la calidad. La superficialidad y la improvisación que generó la temática de la excelencia amaga repetirse con fenómeno de la calidad." (Tomado de Ricardo Riccardi . La cultura de la calidad. Cap. 1. La "Excelencia" ¿Se evaporó?)

\section{BIBLIOGRAFIA}

1. Salud y Gerencia, Enero-Abril $1992 \quad \mathrm{~N}^{\circ}$. Universidad Javeriana.

2. Riccardo Riccardi. La Cultura de la Calidad Total. 1993. Lima. Ediciones Riccardo Riccardi.

3.Educación Permanente del Personal de Salud. OPS \OMS. 1994. Serie DRH N 100.

Rev Med Hered 9(1), 1998 
